In Crescendo, 2017; 8(2): 193-209

Fecha de recepción: 31 de mayo del 2017

Fecha de aceptación: 4 de noviembre del 2017

\title{
DESCUBRIMIENTO DEL ESTILO DE APRENDIZAJE DOMINANTE DE ESTUDIANTES DE LA CARRERA DE TECNOLOGÍA EN ANÁLISIS DE SISTEMAS
}

\author{
DISCOVERY DOMINANT LEARNING STYLE OF STUDENTS \\ STUDYING TECHNOLOGY IN SYSTEMS ANALYSIS
}

Roberto Camana ${ }^{1}$, Rolando Torres ${ }^{2}$

\section{RESUMEN}

La presente investigación tuvo como objetivo, descubrir cuáles son los estilos de aprendizaje dominante de estudiantes de primero a cuarto nivel de la Carrera de Tecnología en Análisis de Sistemas, semestre académico Octubre 2015 - Marzo 2016, con el propósito de mejorar el proceso de enseñanza de estudiantes. Para llevar este estudio, se plantearon los siguientes procesos: Elaboración del estado del arte en torno a estilos de aprendizaje para conocer las diversas metodologías y resultados. El modelo utilizado, es el propuesto por Felder-Silverman, porque, este instrumento se basa en la calidad, confiabilidad y validez, para descubrir el estilo de aprendizaje dominante. Para este estudio se aplicó el Proceso de Extracción de Conocimiento a partir de Datos, con sus etapas: Preprocesamiento, Minería de Datos y Postprocesamiento, con el objetivo de identificar grupos homogéneos dentro de los estudiantes encuestados, por medio de técnicas descriptivas. Se utilizó software libre y de código abierto, Weka, porque permite

1 Master en Minería de Datos, Universidad de Buenos Aires. Ingeniero en Sistemas, Universidad Tecnológica Indomérica. Cursa la Maestría en Informática Educativa, Universidad Técnica de Ambato. Es Docente investigador y Director de la Carrera de Análisis de Sistemas del Instituto Tecnológico Superior Aloasí. Es autor de varios libros, artículos en revistas indexadas y articulista. robertocamana@yahoo.es

2 Especialización Superior en Gestión de la Calidad en la Educación, Universidad Andina Simón Bolívar (en curso). Licenciado en Ciencias de la Educación, mención: Psicología Educativa y Orientación. Es coordinador de la Unidad de Bienestar Estudiantil del Instituto Tecnológico Superior Aloasí. Ha desempeñado funciones de Docente y Orientador vocacional en algunas instituciones de Educación básica, medio y superior. Instituto Tecnológico Superior Aloasí, Ecuador. 
implementar una variedad de algoritmos. El estilo de aprendizaje dominante de los estudiantes de la Carrera de Tecnología en Análisis de Sistemas fue: Activo (ACT) - Visual (VIS) Secuencial (SEC) / - Intuitivo (INT). Esta investigación, es parte de una serie de investigaciones, que lleva el Instituto Tecnológico Superior Aloasí.

PALABRAS ClAVE: Algoritmos, docentes, estilos de aprendizaje, estudiantes, grupos homogéneos, minería de datos educacionales.

\section{ABSTRACT}

This research aimed to discover what styles dominant learning students from first to fourth level of the Career Technology are in Systems Analysis, academic semester October 2015 March 2016 with the aim of improving the teaching of students. To carry this study they raised the following processes: Preparation of state of the art about learning styles to meet the various methodologies and results. The model used is the one proposed by Felder-Silverman, because this instrument is based on the quality, reliability and validity, to discover the dominant learning style. For this study the extraction process was applied Knowledge from Data, with its stages: preprocessing, data mining and post-processing, in order to identify homogeneous groups within the surveyed students, through descriptive techniques. Free software was used and open source, Weka, because it allows implement a variety of algorithms. The dominant style of student learning Career Technology Systems Analysis was: Active (ACT) - Visual (VIS) - Sequential (SEC) / - Intuitive (INT). This research is part of a series of investigations, leading the Institute Technological Highter Aloasí.

KEY WORDS: Algorithms, teachers, learning styles, students, homogeneous groups, educational data mining.

\section{INTRODUCCIÓN}

En el ambiente estudiantil, uno de los procesos que a los estudiantes les permita evidenciar su aprendizaje en clases o fuera de ella es por medio de la realización de sus trabajos o tareas. Sin embargo, Díaz (2012) afirma que este proceso se debe considerar que no todos los estudiantes logran su aprendizaje de una manera satisfactoria, es decir, no todos los estudiantes tienen un mismo estilo de aprendizaje o terminan aprendiendo todos en un mismo nivel.

Pero hay un problema, aún no hemos podido comprender del todo cómo nuestros estudiantes aprenden, tan solo conocemos que existen muchas maneras de aprender, tales como: observando, escuchando, reflexionando, debatiendo y hasta a veces actuando. Pero existen otras, que requieren de lógica y razonamiento para aprender, como: memorizando, visualizando, construyendo analogías y hasta a veces de forma intuitiva. 
Si los estudiantes tienen sus formas de aprendizaje, el docente también tiene su forma de enseñanza, como: leer, debatir, reflexionar, mientras ciertos docentes se centran en la práctica más que la teoría o el análisis de casos. Es decir, todos llegan a un mismo punto, que sus estudiantes sean memoristas o que hayan comprendido algo. Cada estudiante dependerá de la habilidad que tenga para aprender y cuán preparado se encuentre. Además dependerá de los factores, estilos de aprendizaje del estudiante y la enseñanza por parte del docente.

Si los estilos de aprendizaje son tomados en cuenta en el proceso de adquisición de conocimientos James Keefe (1982) menciona que los estilos de aprendizaje son aquellos rasgos cognitivos, afectivos y fisiológicos que operan como posibles indicadores de cómo un individuo promedio percibe, apropia e interactúa o responde a una determinada información.

En dicha apreciación el autor integra los principales aspectos que dan sentido y base a los estilos de aprendizaje, aspectos de orden cognitivo que hacen alusión a la adquisición del conocimiento que mantiene un estudiante en su jornada académica.

Estudios desarrollados por Peter Honey \& Allan Mumford en 1986 acerca de los los estilos de aprendizaje demuestran que los estudiantes presentan los siguientes estilos: Activo el mismo que se implica plenamente en nuevas experiencias; Reflexiva al que le gusta considerar las experiencias y observarlas desde diferentes perspectivas; Teórico quien adapta e integra las observaciones dentro de categorías lógicas y complejas; y Pragmático quien impulsa descubrir el aspecto positivo de las nuevas ideas y aprovechar la primera oportunidad para experimentar, en definitiva aplica en la práctica las ideas adquiridas.

Con estos estudios, Felder y Silverman (1988), desarrollaron su modelo de estilo de aprendizaje, para identificar los más importantes estilos de aprendizaje de estudiantes de ingeniería, con la intención de obtener una base para diseñar estrategias de enseñanza por parte de docentes. Este modelo se subdivide en cuatro dimensiones, es decir cada una está compuesta por dos estilos de aprendizaje opuestos: Activo o Reflexivo, Sensitivo o Intuitivo, Visual o Verbal y Secuencial o Global (Ismaila y Jamaluddina, 2010).

Es así, docentes de la Carrera de Tecnología en Análisis de Sistemas del Instituto Tecnológico Superior "Alosí", ubicado en el Distrito Metropolitano de Quito-Ecuador. Es de vital importancia, conocer cómo aprenden nuestros estudiantes, con la finalidad de encontrar los estilos de aprendizaje predominantes en 
los estudiantes (adecuada estrategia de enseñanza). Incluso, cuando se trate de tutorías, las características de estilos de aprendizaje de estudiantes, al docente, le permitirá formar grupos de aprendizaje homogéneos, para fortalecer intervención educativa (Juárez, Rodríguez y Luna, 2012).

Identificar el estilo de aprendizaje garantizará el avance del proceso de enseñanza, siempre que se conozca el estilo más real o próximo al existente dentro del grupo de estudiantes (Díaz-Ovilla, Rico, Arellano y Guzmán-Zazueta, 2013). Con este antecedente, conocer el estilo de aprendizaje, debe ser tratado de una forma técnica, para ello se plantea la aplicación de técnicas de minería de datos, mediante la técnica de aprendizaje no supervisado, es decir no existe un conocimiento a priori, donde se analicen las relaciones entre variables.

En Ecuador, el tema de minería de datos educacionales, aplicado a la investigación de estilos de aprendizaje dominante en estudiantes, a nivel de Educación Superior Tecnológica, existen pocos estudios realizados al respecto. Nuestro objeto de estudio fueron estudiantes del semestre académico Octubre 2015 - Marzo 2016, de la Carrera de Tecnología en Análisis de Sistemas del Instituto Tecnológico Superior Aloasí.

Sin embargo, existen en la actualidad numerosas investigaciones en torno al análisis de estilos de aprendizaje. En México, los investigadores Costaguta, Menini, Missio y Roldán (2015), utilizaron la técnica de minería de datos, como es el aprendizaje de máquina, para analizar el comportamiento de estudiantes y docentes, para obtener mejores resultados en el proceso enseñanza-aprendizaje en estudiantes de alto rendimiento. En este mismo país, Díaz-Ovilla (2013), aplicaron la estrategia de la predicción para la identificación de estilos de aprendizaje, mediante una técnica de minería de datos, como es la clasificación, que consistió en la discriminación a través de un centroide.

Entre otros métodos utilizados, fue en Argentina, los investigadores Ventura, Gagrildi y Moscoloni (2012), aplicaron la técnica descriptiva de minería de datos, de la prueba Kruskal Wallis, que consiste para contrastar entre las carreras universitarias y edad en intervalos. Además, la prueba U de Mann-Whitney, con el fin de contrastar el género (hombre y mujer), todas estas pruebas para el estudio de las relaciones existentes de estilos de aprendizaje.

Este artículo tuvo como propósito, tras aplicar técnicas de minería de datos educacionales, permitió descubrir el estilo de aprendizaje dominante en estudiantes de la Carrera de Tecnología en Análisis de Sistemas, como instrumento 
de apoyo al Docente en el proceso de enseñanza. Además, avala en el Reglamento Orgánico Interno del ITSA, ROI (2015), artículo 57, literal m, señala “Diseñar la política, filosofía, estrategias e instrumentos de todas las etapas del proceso de enseñanza-aprendizaje, de acuerdo con las normas generales establecidas por el $\mathrm{H}$. Consejo Directivo y las autoridades gubernamentales de la educación superior".

Esta investigación, se enmarca dentro de las líneas de investigación del Instituto Tecnológico Superior Aloasí: Ciencias de la computación, sub línea minería de datos, debido que esta investigación desemboca en un proceso que intenta descubrir patrones en el ámbito educativo en grandes volúmenes de conjuntos de datos. Entre sus métodos aplica el Proceso de Extracción de Conocimiento a partir de Datos.

\section{REFERENTES TEÓRICOS}

\section{MODELOS DE ESTILOS DE APRENDIZAJE}

El término "estilo de aprendizaje" hace referencia, a que cada ser humano tiene su propia forma para aprender. De modo, que se convierte en una estrategia, de acuerdo a lo que desee aprender el estudiante, desarrollando habilidades y destrezas, en conjunto definen su estilo de aprendizaje. Entre los rasgos característicos tenemos, el cognitivo, el afectivo y el fisiológico, que sirven de indicadores para establecer cómo los estudiantes perciben y responden ante un ambiente de aprendizaje. El ámbito cognitivo, consiste en la forma, que los estudiantes logran estructurar contenidos, utilizan conceptos, saben cómo interpretar la información, entre otros. El afectivo, responde a los afectos, tales como, motivacionales y expectativas. Mientras tanto el fisiológico, se relaciona con el género y aspectos biológicos, en el cual tenemos el sueño-vigilia (Woolfolk, 1996).

Bajo este ámbito se han desarrollado varios modelos y teorías sobre estilos de aprendizaje. Para nuestro estudio, se eligió el modelo de estilos de aprendizaje de Felder-Silverman, porque, se basa en la calidad de su estudio usando instrumentos con una alta validez del instrumento, que provee la identificación de estilos de aprendizaje en estudiantes de la Carrera de Tecnología en Análisis de Sistemas. Además, el cuestionario ha sido validado y probado por Felder (2005), lo que proporciona un mayor soporte y grado de confiabilidad, que otros modelos que no lo tienen (Zatarain, 2011). 
Los autores del modelo, Felder y Silverman (1988), se centran en tres aspectos, el primero, en el estilo de aprendizaje significativo en la educación de ingeniería en sistemas; el segundo, el estilo de aprendizaje preferido por los estudiantes y las estrategias que receptan los estudiantes, cuyos estilos de aprendizaje, no son abordados por los métodos de enseñanza de ingeniería formal. Los autores proponen, cuatro dimensiones para el análisis, entre las que constan: Procesamiento (Activo o Reflexivo), Percepción (Sensorial o Intuitivo), representación (Visual o Verbal) y compresión (Secuencial o global). A continuación se presentan en la tabla 1 de los descriptores.

Tabla 1

DESCRIPTORES DE LOS CUATRO ESTILOS DE APRENDIZAJE UTILIZADOS

\begin{tabular}{|l|l|l|l|}
\hline \multicolumn{1}{|c|}{ Procesamiento } & \multicolumn{1}{|c|}{ Percepción } & Representación & \multicolumn{1}{c|}{ Comprensión } \\
\hline $\begin{array}{l}\text { ¿Cómo prefiere } \\
\text { adquirir } \\
\text { información? }\end{array}$ & $\begin{array}{l}\text { ¿Qué tipo de } \\
\text { información } \\
\text { prefiere recibir? }\end{array}$ & $\begin{array}{l}\text { ¿A través de qué } \\
\text { vía sensorial capta } \\
\text { la información? }\end{array}$ & $\begin{array}{l}\text { iDe qué modo } \\
\text { facilita el } \\
\text { entendimiento } \\
\text { de contenidos }\end{array}$ \\
\hline
\end{tabular}

A continuación, se ejemplariza cada uno de los estilos de aprendizaje:

El aprendizaje Activo: Retiene y comprende mejor una nueva información cuando hace algo activo con ella, es decir aprende ensayando y trabajando con otros. Reflexivo: Retiene y comprende una nueva información pensando y reflexionando sobre ella, es decir aprende meditando, pensando y trabajando solos.

El aprendizaje Sensitivo: Son concretos, prácticos, hechos reales, resuelven problemas siguiendo procedimientos muy bien establecidos o estructurados MEA (2004), es decir prefieren memorizar hechos con facilidad. Intuitivo: Son conceptuales, innovadores, teóricos, es decir aprende rápidamente nuevos conceptos, trabaja bien con abstracciones y fórmulas matemáticas.

El aprendizaje Visual: Tiende a la obtención de información por representaciones visuales, porque recuerda mejor lo que ve, por medio de diagramas de flujo, símbolos, etc. Verbal: Prefiere obtener información en forma escrita, es decir recuerda mejor lo que lee o escucha.

El aprendizaje Secuencial: Tiende a solucionar problemas, mediante caminos por pequeños pasos lógicos MEA (2004), es decir aprende en pequeños pasos 
incrementales. Global: Tiende a aprender nuevo material de pronto visualiza la totalidad, es decir aprende a grandes saltos, porque resuelven problemas complejos rápidamente.

\section{MÉTODO DE DESARROLLO}

La existencia de grandes volúmenes de datos, contenidos en gigantescas bases de datos, en muchos casos excede de nuestra capacidad humana para poder analizarlos y obtener información útil, realidad que viven día a día muchas organizaciones en el mundo. La búsqueda en la inmensidad de datos se dedican expertos para encontrar patrones de comportamiento que ayuden anticipar nuestras decisiones (Camana, 2013). En este sentido, las decisiones, se toman muchas veces en base a la intuición y las experiencias previstas en la información almacenada. De modo, se intenta solucionar mediante el Proceso de Extracción de Concomimiento, por sus siglas en inglés KDD (Knowledge Data Discovery), este proceso contempla tres etapas: Preprocesamiento, Minería de datos y Postprocesamiento. En la Figura 1, se muestra las etapas del KDD:

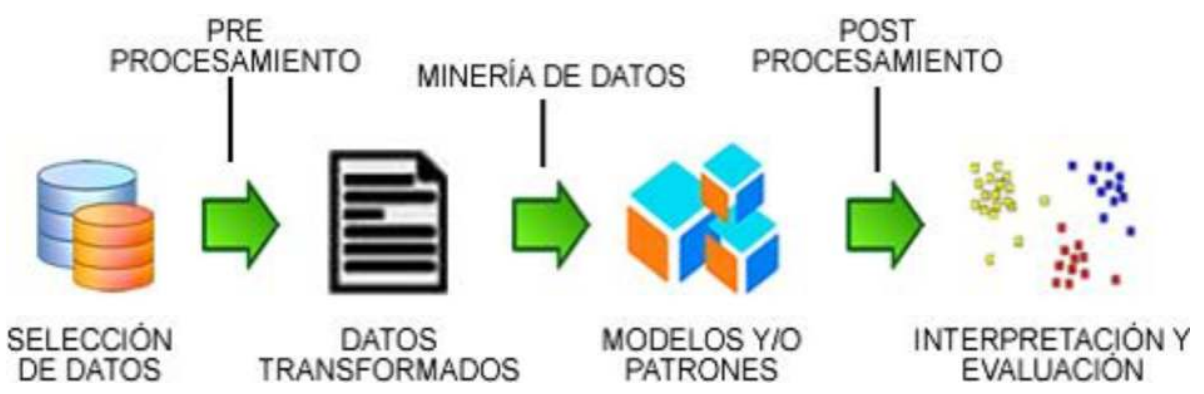

Figura 1. Proceso KDD.

La etapa de Preprocesamiento, consiste en preparar los datos, para que puedan ser utilizados en la siguiente etapa de la minería de datos, dentro de esta etapa, según Han y Kamber (2001), mencionan los siguientes procesos: Selección, que consiste en la recopilación e integración de datos de determinadas fuentes útiles de información, con la finalidad de seleccionar variables relevantes para el estudio. La Limpieza de datos, con el fin de eliminar la mayor cantidad de datos erróneos e inconsistentes (limpieza) e irrelevantes. La Transformación de datos, con la finalidad de reducir el almacén de datos, por ejemplo eliminando caracte- 
rísticas redundantes. En esta etapa es importante, por cuanto es el procesamiento del almacén de datos en busca de la calidad de los datos, de tal forma permitirá lograr la calidad del conocimiento que se descubra (Pérez y Santín, 2007).

En la etapa de Minería de Datos, se decide, qué tarea se va a realizar, por ejemplo agrupar o clasificar. Se eligió la técnica de clasificación descriptiva, según Pérez y Santin (2007), en esta técnica no existen variables dependientes ni independientes, y tampoco un modelo previo para los datos. En cambio la técnica predictiva, específica un modelo de datos para los datos de un conocimiento teórico previo. De las dos técnicas antes mencionadas, la más apropiada para esta investigación fue la técnica descriptiva, por cuanto se crean modelos automáticos a partir del reconocimiento de patrones del conjunto de datos.

La etapa de Postprocesamiento, consiste en la evaluación e interpretación de resultados (patrones) y evaluación de modelos, que es analizada por los expertos. De ser necesario, se vuelve a las fases anteriores para una nueva iteración. Para finalmente, una fase de difusión, con el fin socializar el nuevo conocimiento con todos los demás usuarios.

\section{DESARROLLO DE LA INVESTIGACIÓN}

Los docentes de la Carrera de Tecnología en Análisis de Sistemas, consideramos de eminente utilidad, determinar las características del perfil del estudiante en cuanto a su estilo de aprendizaje. De modo, que nos permitirá adecuar de mejor forma la estrategia de enseñanza a nuestros estudiantes. En este contexto, la principal fuente de datos para el desarrollo de esta investigación, fueron obtenidos de estudiantes.

La Carrera de Tecnología en Análisis de Sistemas, en el periodo Octubre 2015 - Marzo 2016, contó con 37 mujeres y 46 hombres, con un total de 83 estudiantes, esta muestra representa el $51 \%$, del total del universo de estudiantes matriculados en el mencionado periodo académico.

De esta manera, se obtuvo un insumo de datos, tras aplicar a cada estudiante el test propuesto por Felder y Silverma, mediante una encuesta en línea y a través de Formularios de Google, que consta de cuarenta y cuatro preguntas, con dos opciones de respuesta (A y B), donde cada estudiante encuestado debe elegir una sola opción de respuesta. En la Figura 2, se muestra la planilla de datos de la encuesta. 


\begin{tabular}{|c|c|c|c|c|c|c|c|c|c|c|c|c|c|c|c|}
\hline 4 & A & B & C & D & $E$ & $\mathrm{~F}$ & G & $\mathrm{H}$ & 1 & $\mathrm{~J}$ & $\mathrm{~K}$ & L & M & $\mathrm{N}$ & 0 \\
\hline 1 & APELLIDOS_Y_NOMBRES & P1 & P2 & P3 & P4 & P5 & P6 & P7 & P8 & P9 & P10 & P11 & P12 & P13 & P14 \\
\hline 2 & ACHIG ANDRADE HENRY DAVID & A & $B$ & A & $B$ & $B$ & B & $B$ & A & A & A & $B$ & $B$ & A & 8 \\
\hline 3 & AGUIAR UZHCAFRANKLIN EDUARDO & A & A & A & A & A & $B$ & A & A & $B$ & A & $B$ & A & A & A \\
\hline 4 & AGUILAR MORALES KRISTOPHER ANDRES & A & $B$ & A & A & $B$ & A & A & A & A & A & A & A & A & $B$ \\
\hline 5 & AIMARA OLMEDO JENIFFER LIZETH & A & A & A & A & A & $B$ & $B$ & A & $B$ & $B$ & $B$ & A & A & A \\
\hline 6 & ALLAICAALLAICADIEGO FERNANDO & A & B & A & B & A & A & A & A & $B$ & A & A & A & A & 8 \\
\hline 7 & ALOMOTO CATOTA ADRIAN GEONANNY & A & $B$ & A & A & A & A & A & 8 & A & $A$ & A & A & A & A \\
\hline 8 & ALTA CALAPI CARLOS ANTONIO & A & A & $B$ & B & A & $B$ & A & A & A & A & A & A & A & A \\
\hline 9 & ALTAMIRANO TAYUPANTA BEATRIZ PILAR & A & A & $B$ & $B$ & $B$ & A & $B$ & B & A & $B$ & $B$ & $B$ & A & A \\
\hline 10 & ALVARO FARINANGO JORGE LUIS & A & A & $B$ & B & B & A & $B$ & 8 & A & A & A & $B$ & A & A \\
\hline 11 & AMAGUANA CAIZA JOHNNY EDISON & A & A & B & A & A & A & A & $B$ & A & A & A & A & A & A \\
\hline 12 & ANGUETALOPEZ WLLMER HENRY & A & $B$ & A & B & A & A & $B$ & A & A & A & A & A & A & A \\
\hline 13 & ARELLANO ARELLANO ALEX OLMEDO & A & B & A & B & B & A & B & B & A & A & A & B & A & $B$ \\
\hline 14 & ASHQUI ACURIO MARIA SABINA & $B$ & A & B & A & $B$ & A & A & A & A & $A$ & $B$ & B & A & A \\
\hline 15 & BRAVO CORDOVA WILSON ADRIAN & A & $B$ & A & $A$ & A & A & A & B & $B$ & $B$ & A & A & $A$ & $B$ \\
\hline 16 & BRIONES PARAPI DANIEL AGUSTIN & A & A & A & B & A & A & A & A & A & A & A & A & $B$ & A \\
\hline 17 & CAIZA ALMACHE MARTHA YESENIA & A & A & A & A & A & A & A & A & A & $B$ & A & A & A & A \\
\hline 18 & CAIZA CAIZA SANTIAGO PAUL & A & $B$ & $B$ & A & $B$ & $B$ & A & $B$ & A & A & A & A & A & 8 \\
\hline 19 & CAIILEMA CHARICANDO SEGUNDO NICOLAS & A & B & A & A & B & A & B & B & A & A & $B$ & A & A & $B$ \\
\hline 20 & CASTILLO CHAVEZ ANDREA MICHELLEE & A & $B$ & A & $B$ & $B$ & A & A & B & A & $B$ & A & A & A & $B$ \\
\hline 21 & CHAMORRO LOPEZ JOSE ANDRES & A & B & A & A & A & B & B & B & A & A & B & A & A & A \\
\hline 22 & CHANGO AVLES JOHN JARO & A & A & B & A & A & $B$ & $B$ & A & A & A & A & A & $B$ & 8 \\
\hline 23 & CHAUCA CHAVARRIA SOFIA ARACELYY & B & A & A & A & $B$ & A & B & 8 & B & A & A & A & A & 8 \\
\hline 24 & CHICAIZA EGAS HUGO JAVIER & $B$ & A & A & $B$ & A & A & A & $B$ & A & $B$ & A & $B$ & A & $B$ \\
\hline 25 & CHIGUANO YAUTIBUG DAVID EDUARDO & A & A & A & $B$ & $B$ & A & $B$ & $B$ & A & $A$ & A & $B$ & A & $B$ \\
\hline
\end{tabular}

Figura 2. Datos obtenidos a través del formulario de Google.

Con la información recolectada a través de la encuesta en línea, generó una base de datos almacenada en una plantilla de Excel, para posteriormente ser convertida a un archivo con extensión .arff (creado por la Universidad de Weikato). Según Witten y Frank (1999), este archivo fue utilizado por Weka, que sirvió para el Proceso de Descubrimiento del Conocimiento, con sus etapas Preprocesamiento, Minería de Datos y Posprocesamiento.

En la etapa de Preprocesamiento, el proceso de limpieza de datos, se realizaron correcciones manuales, como la verificación de apellidos y nombres de estudiantes, para ello se constató con listas de matriculados proporcionado por Secretaría del Instituto Tecnológico Superior Aloasí. Dado que el archivo donde se almacenó los datos es generado por la encuesta a través de un Formulario de Google, por lo tanto, no fue necesario realizar tareas de integración ni de transformación. Cabe destacar, que el único proceso utilizado fue el de reducción de datos, que consistió en la eliminación de atributos: Fecha, hora, género, carrera, nivel y modalidad, por cuanto estos atributos resultan irrelevantes para alcanzar los objetivos del Proceso de Descubrimiento del Conocimiento. En la Figura 3, se muestra la conversión del archivo de Excel a formato .arff en software Weka.

Descubrir los estilos de aprendizaje dominante en estudiantes de la Carrera de Tecnología en Análisis de Sistemas, es un reto de la minería de datos educacionales. Siendo este un proceso de análisis, que permitirá al docente mejorar su estrategia de enseñanza a sus estudiantes. Por lo tanto, se presentan cinco pre- 


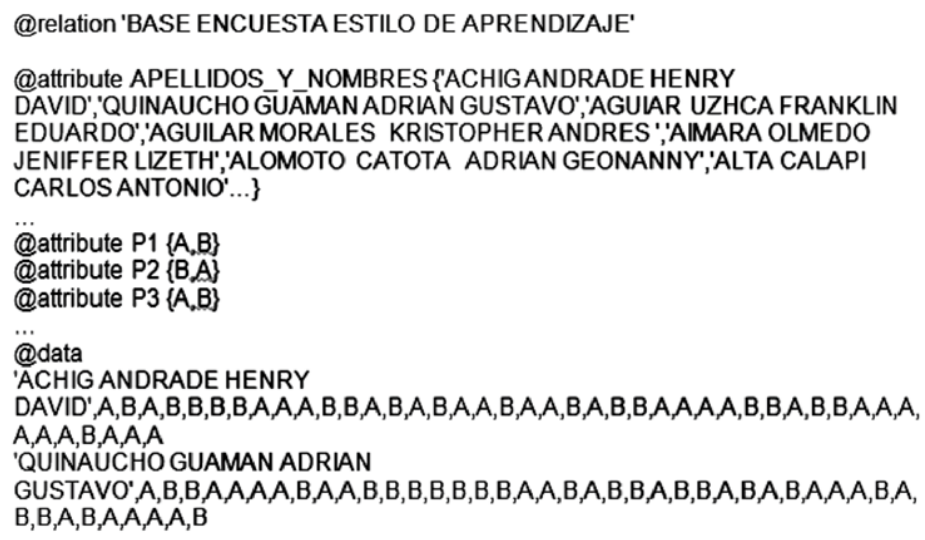

Figura 3. Archivo .arff generado por el software Weka.

guntas planteadas por Han y Kamber (2001), para realizar el descubrimiento de estilos de aprendizaje. Corresponde a la segunda etapa (Minería de Datos) del Proceso de Extracción de Conocimiento, entre las que constan las siguientes:

Se decidió trabajar con el conjunto de datos resultante de la primera etapa (Preprocesamiento) del Proceso de Extracción del Conocimiento, porque estos atributos son los más relevantes para lograr descubrir un grupo dominante de estudiantes según su estilo de aprendizaje encontrado. Se utilizaron los atributos: Nombre_apellido, para identificación de los estudiantes y 44 preguntas con sus dos tipos de respuestas "A" y "B", test propuesto por Felder y Silverma.

La técnica descriptiva de minería de datos es la más adecuada, por cuanto se aplicó el análisis de clustering, con el fin de identificar grupos homogéneos dentro de los estudiantes encuestados. Para este estudio se utilizó Weka, software libre y de código abierto, que implementa una variedad de algoritmos y herramientas útiles para tareas de minería de datos.

Se utilizó cluster con su algoritmo FarthestFirst, porque al tratarse de un problema de k centros (grupos), se pretende obtener la máxima distancia entre una tupla (estudiante) y su centroide sea la mínima Chaudhuri, Garg y Ravi (1998). De modo, que para descubrir el estilo de aprendizaje dominante de los estudiantes, se decidió generar dos clusters.

Como se puede apreciar a continuación los resultados, no se necesita ampliar el número de clusters, porque el mayor número de tuplas (73) están incluidas en el primer cluter. 
Scheme:weka.clusterers.FarthestFirst-N2 -S 150

Relation: ESTILOS_APRENDIZAJE_ANALISIS_SISTEMAS

Instances: 83

Attributes: 45

APELLIDOS_Y_NOMBRES

P1

$\mathrm{P} 2$

$\mathrm{P} 3$

$\cdots$

P44

Test mode:evaluate on training data

$===$ Model and evaluation on training set $===$

FarthestFirst

Cluster centroids:

Cluster 0

AB ABAAAAB AAAAB BABAAAAB AAB AAB B BAAAB ABAB ABAAAA

Cluster 1

BAB B AB ABAB BAAB B BAAB BAAAB B BABABABABAAB AB AB B B B

Time taken to build model (full training data): 0 seconds

$===$ Model and evaluation on training set $===$

Clustered Instances

$0 \quad 73(88 \%)$

$110(12 \%)$

A partir de las características definidas en los estilos de aprendizaje por Felder y Silverman y sus posibles respuestas de las cuarenta y cuatro preguntas del test propuesto por autores antes mencionados. Se muestran a continuación en la Tabla 2, a fin de identificar cómo aprenden los estudiantes, de acuerdo alguno de las características del modelo de aprendizaje.

A partir del conocimiento obtenido, se decidió reemplazar cada uno de los atributos en los dos grupos o centroides, ejecutados por el software libre Weka, como se indica en la Tabla 2. Efectuado el reemplazo, quedó expresada cada una de las respuestas obtenidas: 
Tabla 2

ESTILOS DE APRENDIZAJE Y SUS RESPUESTAS

\begin{tabular}{|c|c|c|}
\hline Estilos de aprendizaje & ID & Respuestas \\
\hline Activo & ACT & $\begin{array}{l}P 1=A, P 5=A, P 9=A, P 13=A, P 17=A, P 21=A, \\
P 25=A, P 29=A, P 33=A, P 37=A, P 41=A,\end{array}$ \\
\hline Reflexivo & REF & $\begin{array}{l}P 1=B, P 5=B, P 9=B, P 13=B, P 17=B, P 21=B, \\
P 25=B, P 29=B, P 33=B, P 37=B, P 41=B\end{array}$ \\
\hline Sensitivo & SEN & $\begin{array}{l}P 2=A, P 6=A, P 10=A, P 14=A, P 18=A, P 22=A, \\
P 26=A, P 30=A, P 34=A, P 38=A, P 42=A,\end{array}$ \\
\hline Intuitivo & INT & $\begin{array}{l}P 2=B, P 6=B, P 10=B, P 14=B, P 18=B, \\
P 22=B, P 26=B, P 30=B, P 34=B, P 38=B, P 42=B,\end{array}$ \\
\hline Visual & VIS & $\begin{array}{l}P 3=A, P 7=A, P 11=A, P 15=A, P 19=A, \\
P 23=A, P 27=A, P 31=A, P 35=A, P 39=A, P 43=A,\end{array}$ \\
\hline Verbal & VER & $\begin{array}{l}P 3=B, P 7=B, P 11=B, P 15=B, P 19=B, \\
P 23=B, P 27=B, P 31=B, P 35=B, P 39=B, P 43=B,\end{array}$ \\
\hline Secuencial & SEC & $\begin{array}{l}P 4=A, P 8=A, P 12=A, P 16=A, P 20=A, \\
P 24=A, P 28=A, P 32=A, P 36=A, P 40=A, P 44=A,\end{array}$ \\
\hline Global & GLO & $\begin{array}{l}P 4=B, P 8=B, P 12=B, P 16=B, P 20=B, P 24=B, \\
P 28=B, P 32=B, P 36=B, P 40=B, P 44=B\end{array}$ \\
\hline
\end{tabular}

A partir del conocimiento obtenido, se decidió reemplazar cada uno de los atributos en los dos grupos o centroides, ejecutados por el software libre Weka, como se indica en la Tabla 2. Efectuado el reemplazo, quedó expresada cada una de las respuestas obtenidas:

\section{Centroide (Cluster 0)}

ACT, INT, VIS, GLO, ACT, SEN, VIS, SEC, REF, SEN, VIS, SEC, ACT, INT, VER, SEC, REF, SEN, VIS, SEC, ACT, INT, VIS, SEC, REF, SEN, VIS, GLO, REF, INT, VIS, SEC, ACT, INT, VIS, GLO, ACT, INT, VIS, GLO, ACT, SEN, VIS, SEC

\section{Centroide (Cluster 1):}

REF, SEN, VER, GLO, ACT, INT, VIS, GLO, ACT, INT, VER, SEC, ACT, INT, VER, GLO, ACT, SEN, VER, GLO, ACT, SEN, VIS, GLO, REF, INT, VIS, GLO, ACT, INT, VIS, GLO, ACT, INT, VIS, SEC, REF, SEN, VER, SEC, REF, INT, VER, GLO 
Sustituido los atributos por el respectivo estilo de aprendizaje, representado por cada centroide, se procedió a contar la cantidad de concurrencias, por cada estilo de aprendizaje, a continuación se obtuvo los siguientes resultados:

Centroide (Cluster 0$)$ :

$\begin{array}{ll}\text { ACT }=7 & \text { REF }=4 \\ \text { SEN }=5 & \text { INT }=6 \\ \text { VIS }=10 & \text { VER }=1 \\ \text { SEC }=7 & \text { GLO }=4\end{array}$

Este centroide representa el estilo de aprendizaje: Activo (ACT) - Visual (VIS) - Secuencial (SEC) / Intuitivo (INT), siendo el dominante en la población de estudiantes encuestados de la Carrera de Tecnología en Análisis de Sistemas.

Centroide (Cluster 1$)$ :

$\begin{array}{ll}\text { ACT }=7 & \text { REF }=4 \\ \text { SEN }=4 & \text { INT }=7 \\ \text { VIS }=5 & \text { VER }=6 \\ \text { SEC }=3 & \text { GLO }=8\end{array}$

En cambio en este centroide representa el estilo de aprendizaje: Activo (ACT)/Intuituivo (INT) - Verbal (VER) - Global (GLO) .

Se optó por una visualización generada por la herramienta Weka, con su opción Cluster (FarthestFirst), porque muestran los clusters resultantes en función al número de instancias por registros de estudiantes, como se observa en la Figura 4.

En términos generales, aproximadamente el $88 \%$ del cluster 0 (instancias de color azul), de estudiantes encuestados a través del test propuesto por Felder y Silverman es supremamente dominante al cluster 1, que tan solo llega al 12\% (instancias de color rojo).

\section{RESULTADOS Y DISCUSIÓN}

En la práctica diaria del docente y estudiantes en el aula, permite establecer una relación entre los estilos de aprendizaje y la forma de enseñar de los docentes. De acuerdo a los resultados obtenidos, se propone algunas estrategias de enseñanza, en base al perfil dominante obtenido de los estudiantes a través de sus estilos de aprendizaje, por lo tanto se sugiere lo siguiente: 


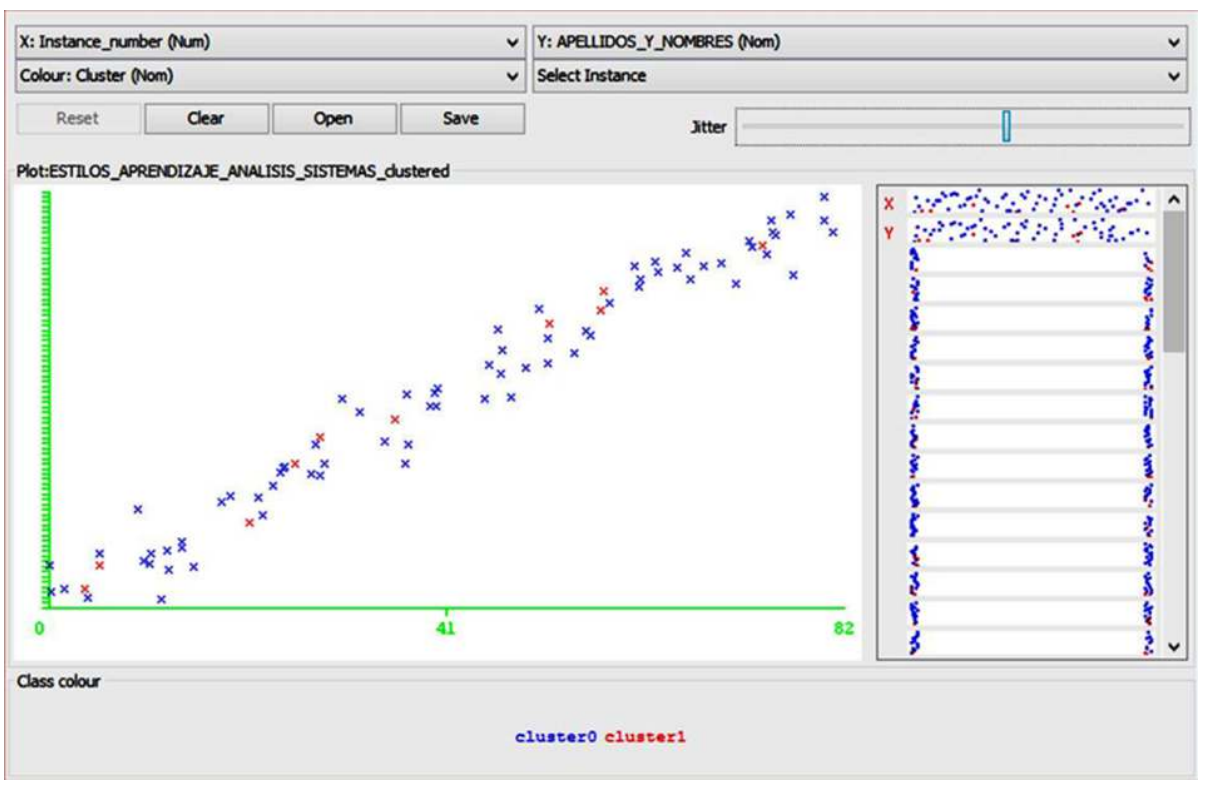

Figura 4. Cluster resultantes.

En la primera dimensión (más Activo que Reflexivo), se sugiere que la participación activa del estudiante sea opinando, reflexionando y actuando. Las asignaturas que presentan estas características son: Comunicación oral y escrita, Realidad nacional, Metodología de la investigación.

Es necesario que la participación activa, sea conveniente, que el docente alterne la lectura de material bibliográfico y clases expositivas, estas características se pueden aplicar en todas las asignaturas.

En la segunda dimensión (Intuitivo predomina a lo Sensorial), su tendencia es hacia lo innovador, comprenden rápidamente nuevos conceptos, odian la repetición, se caracterizan por trabajar con abstracciones (lógica de programación) y fórmulas matemáticas, además no son memoristas o realizan cálculos rutinarios.

En la tercera dimensión (Predominio de lo Visual ante lo Verbal), la información recibida por los estudiantes debe ser presentada de forma visual, es decir prefieren para su aprendizaje de imágenes, diagramas de flujos. Esta característica es propicia para todas las asignaturas de programación I, programación estructurada y programación orientada a objetos, por cuanto van acompañadas de explicaciones de procesos, algoritmos complejos con diagramas de flujos. 
En cuanto a matemática general, matemáticas I y II, al igual que la estadística y probabilidades, es recomendable que las funciones matemáticas complejas deben hacerlo a través de programas informáticos, software especializado (simuladores) en matemáticas y estadística con capacidades de ilustraciones gráficas.

En la cuarta dimensión (Secuencia más que Global), se recomienda que la información proporcionada al estudiante, tenga un progreso en su aprendizaje, es decir implica procedimiento secuencial, por medio de pequeños pasos incrementales, esta característica se puede presentar en asignaturas de secuencias, por ejemplo Optativa I (Proyecto de vida), optativa II, (Desarrollo del pensamiento).

Cuando se trata de solucionar un problema, tienen que seguir caminos por pequeños pasos lógicos. Es decir, seguir una metodología de desarrollo de un programa informático (Análisis, diseño, implementación, ejecución, mantenimiento).

\section{CONCLUSIONES}

La aplicación de la minería de datos educaciones, mediante el Proceso de Extracción del Conocimiento, por sus siglas en inglés KDD (Knowledge Data Discovery), permitió obtener un alto grado de homogeneidad, en el estilo de aprendizaje de los estudiantes de la Carrera de Tecnología en Análisis de Sistemas del Instituto Superior Tecnológico Aloasi. El cluster generado permitió identificar el estilo de aprendizaje Activo (ACT) - Visual (VIS) - Secuencial (SEC) / - Intuitivo (INT).

Se propone un planteamiento de lineamientos generales iniciales para las asignaturas de especialización de la Carrera de Análisis de Sistemas, con la finalidad de encajar el estilo de enseñanza del docente, de acuerdo al estilo de aprendizaje dominante de los estudiantes. Cabe recalcar que los lineamientos planteados deberán ser particularizados para las demás asignaturas. Para validar los resultados obtenidos, se propone implementar en los estudiantes de la asignaturas: Ofimática II, Programación Orientada a Objetos I, Programación I, de la Carrera de Tecnología en Análisis de Sistemas, en el semestre académico (Mayo-Septiembre 2016). Siguientes procedimientos investigativos por otros docentes el tema de estilos de aprendizaje.

Existen educadores que dan prioridad a la evaluación del conocimiento y otros a la evaluación del proceso de aprendizaje; desde cualquier punto de vista la 
metodología de trabajo de cada docente es sin igual, lo importante en definitiva es el aprendizaje adquirido por los estudiantes mediante la incorporación de procesos de enseñanza que el docente utiliza, las herramientas brindadas en este estudio nos permitirán entender las formas de adquisición de los conocimientos.

Esta herramienta de trabajo en el aula debe servir como proceso que acompañamiento y refuerzo pedagógico, respetando el estilo de aprendizaje de los estudiantes, distinguiendo las diferencias individuales que presentan nuestros educandos de tal manera que lo vean como un proceso de desarrollo de sus capacidades y no como un proceso punitivo.

A partir de este trabajo, se sugiere nuevas investigaciones, por cuanto la forma automatizada para obtener información sobre estilos de aprendizaje de los estudiantes, procesarlas y analizarlas, permitirá obtener diversas herramientas de cómo aprenden nuestros estudiantes para la toma de decisiones en el aula de clases.

\section{AGRADECIMIENTOS}

La presente investigación, no hubiera sido posible con el apoyo brindado por las autoridades del Instituto Tecnológico Superior Aloasí, estudiantes de los primeros, segundos y terceros cursos de la Carrera de Tecnología en Análisis de Sistemas, por su valioso aporte de información en los test administrados.

\section{REFERENCIAS BIBLIOGRÁFICAS}

Camana, R. (2013). Generar Conocimiento el Pensar y Hacer. En R. Camana, Digitalizando nuestra vida cotidiana (pp. 73, 74). Ecuador: Su libro.

Chaudhuri, S., Garg, N., y Ravi, R. (1998). “The p, Neighbor k, Center Problem”, en Information Processing Letters, vol. 65, pp. 131, 134.

http://citeseer.ist.psu.edu/chaudhuri98pneighbor.html

Costaguta, R., Menini, M., Missio, D., Roldán, A., Santana, Mansilla, P., Lescano, G., Yanacon, D. y Concha, E. (2015). Potenciando el Aprendizaje Colaborativo Soportado por Computadora: Algunas ideas traducidas en acciones, Seminario "Sistemas y ambientes educativos, SAMBEO 2015”. Seminario llevado a cabo en México D.F., México.

Díaz, E. (2012). Estilos de aprendizaje. Revista EIDOS, 5(1), 5, 11. Recuperado de http://www.ute.edu.ec/revistas/1/articulos/66ab7790, 1068, 4ab5, 8b3f, 56a4925af3bd.pdf

Díaz, Ovalle, C., Rico, A., Arellano, A. y Guzmán, Zazueta, A. (2013). Estrategia para detectar estilos de aprendizaje usando la técnica de particiones. Revista de Estilos de Aprendizaje, 11(12), 1, 14. Recuperado de http://learningstyles.uvu.edu/index.php/jls/article/view/6/2 
Felder, R. y Silverman, L. (1988). Learning and Teaching styles in engineering education. Engineering Education, 78(7), 674, 681.

Felder, R. y Spurlin, J. (2005). Applications, reliability and validity of the index of learning styles. International Journal of Engineering Education, 21(1), 103-112.

Felder, R, y Silverman, L (1988). Estilos de aprendizaje y de enseñanza en la educación de ingeniería, 78 (7), 674, 681. Recuperado de:

http://www4.ncsu.edu./unity/lockers/users/f/felder/public/Papers/LS,1988.pdf

Han, J. y Kamber, M. (2001). Data Mining: Concepts and Techniques. USA, Academic Press.

Honey, P. \& Mumford, A. (1986) Using our learning styles. United Kingdom. The Open University.

Instituto Tecnológico Superior Aloasí (2015, 1 de julio). Reglamento Orgánico Interno (ROI). Recuperado de http://www.itsa.edu.ec/reglamento_organico.pdf

Ismaila, A., Hussaina, R. y Jamaluddina, S. (2010). Assessment of students' learning styles preferences in the faculty of science, Tishreen University, Syria. Procedia Social and Behavioral Sciences, 2, 4087, 4091.

Juárez, C. S, Rodríguez, G. y Luna, E. (2012). El cuestionario de estilos de aprendizaje chaea y la escala de estrategias de aprendizaje acra como herramienta potencial para la tutoría académica. Revista de Estilos de Aprendizaje, 10(10), 148, 171.

Keefe. James. (1979) Estudiante estilos de aprendizaje, México: Asociación Nacional de Secundaria Directores de Escuelas.

Pérez, C., y Santín, D. (2007). Minería de datos. Técnicas y herramientas, Madrid: Thomson

Secretaria de Educación Pública (2004, 12 de diciembre). Manual de estilos de aprendizaje (MEA). Recuperado de:

$<$ http://biblioteca.ucv.cl/site/colecciones/manuales_u/Manual_Estilos_de_Aprendizaje_2 004.pdf $>$

Ventura, A., Gagrildi, R. y Moscoloni, N. (2012). Estudio descriptivo de los estilos de aprendizaje de estudiantes universitarios argentinos.Revista de Estilos de Aprendizaje, 5(9), 71, 84. Recuperado de http://learningstyles.uvu.edu/index.php/jls/article/view/94/59

Woolfolk, A. (1996). Psicología Educativa, Ed. Prentice, Hall, México, 1996.

Witten, I. y Frank, E. (1999). Data Mining: Practical machine, learning tools and techniques with Java implementations. USA, Morgan Kauffmann Publishers.

Zatarain Cabada, R. y M. L. Barrón Estrada (2011). Herramienta de autor para la identificación de estilos de aprendizaje utilizando mapas auto-organizados en dispositivos móviles. Revista electrónica de investigación educativa, 13(1), 43-55. 